Canad. Math. Bull. Vol. 21 (1), 1978

\title{
COEXISTENCE OF SOME B.I.B. DESIGNS BY
}

K. N. MAJINDAR

1. Summary. In this paper we have shown that the existence of some balanced incomplete block (b.i.b. for abbreviation) designs implies the existence of some others in some cases. We have here established the following theorems.

THEOREM 1. If there exists a symmetric b.i.b. design with parameters $v, b, r, k, \lambda$, (where $v=b, r=k$ ), then there exists $a$ b.i.b. design with parameters,

$v^{\prime}=v, \quad b^{\prime}=\left(\begin{array}{l}v \\ 2\end{array}\right), \quad r^{\prime}=r(v-r), \quad k^{\prime}=2(r-\lambda)$,

$$
\lambda^{\prime}=(r-\lambda)(2 r-2 \lambda-1)
$$

THEOREM 2. If there exists a symmetric b.i.b. design with parameters $v, b, r, k, \lambda$, (where $v=b, r=k$ ), then there exists $a$ b.i.b. design with parameters,

$$
v^{\prime}=v, \quad b^{\prime}=\left(\begin{array}{l}
v \\
2
\end{array}\right), \quad r^{\prime}=\left(\begin{array}{c}
v-r \\
2
\end{array}\right), \quad k^{\prime}=v-2 r+\lambda, \quad \lambda^{\prime}=\left(\begin{array}{c}
v-2 r+\lambda \\
2
\end{array}\right) .
$$

THEOREM 3. If there are two b.i.b. designs, one with parameters $v, b, r, k, \lambda$, and the other with parameters $v^{\prime}=k, b^{\prime}, r^{\prime}, k^{\prime}, \lambda^{\prime}$, then there exists $a$ b.i.b. design with parameters,

$$
v^{\prime \prime}=v, \quad b^{\prime \prime}=b b^{\prime}, \quad r^{\prime \prime}=r r^{\prime}, \quad k^{\prime \prime}=k^{\prime}, \quad \lambda^{\prime \prime}=\lambda \lambda^{\prime} .
$$

THEOREM. 4. If there exists $a$ b.i.b. design with parameters $v, b, r, k, \lambda$, then there exists a b.i.b. design with parameters,

$$
v^{\prime \prime}=v, \quad b^{\prime \prime}=b k, \quad r^{\prime \prime}=r(k-1), \quad k^{\prime \prime}=k(\lambda-1), \quad \lambda^{\prime \prime}=\lambda(k-2) .
$$

THEOREM 5. If there exists a resolvable b.i.b. design with parameters $v, b, r, k, \lambda$, (where $v=a$ multiple of $k$, say $n k$ and then $b=n r$ ) and a resolvable b.i.b. design with parameters $v^{\prime}, b^{\prime}, r^{\prime}, k^{\prime}, \lambda^{\prime}$, (where $v^{\prime}=k=n^{\prime} k^{\prime}$ ), then there exists a resolvable b.i.b. design with parameters

$$
v^{\prime \prime}=v, \quad b^{\prime \prime}=b b^{\prime}, \quad r^{\prime \prime}=r r^{\prime}, \quad k^{\prime \prime}=k^{\prime}, \quad \lambda^{\prime \prime}=\lambda \lambda^{\prime} .
$$

THEOREM 6. If there exists a resolvable b.i.b. design with parameters $v, b, r, k, \lambda$, where $k$ is even and $\geq 4$, then there exists a resolvable b.i.b. design with parameters

$$
v^{\prime \prime}=v, \quad b^{\prime \prime}=b\left(\begin{array}{c}
k \\
k / 2
\end{array}\right), \quad r^{\prime \prime}=r\left(\begin{array}{c}
k-1 \\
(k-2) / 2
\end{array}\right), \quad k^{\prime \prime}=k / 2, \quad \lambda^{\prime \prime}=\lambda\left(\begin{array}{c}
k-2 \\
(k-4) / 2
\end{array}\right) .
$$

Received by the editors May 10, 1976. 
2. B.i.b. designs associated with a symmetric b.i.b. design. Given a symmetric b.i.b. design with parameters $v, b, r, k, \lambda$, (where $v=b, r=k$ ). It was shown by Bose that associated with this design are two b.i.b. designs ("the residual designs") with parameters

$$
v^{\prime}=k, \quad b^{\prime}=b-1, \quad r^{\prime}=r-1, \quad k^{\prime}=\lambda, \quad \lambda^{\prime}=\lambda-1
$$

and

$$
v^{\prime \prime}=v-k, \quad b^{\prime \prime}=b-1, \quad r^{\prime \prime}=r, \quad k^{\prime \prime}=k-\lambda, \quad \lambda^{\prime \prime}=\lambda .
$$

Recently Vanstone [1] has proved the following theorem.

THEOREM (Vanstone). Given a symmetric b.i.b. design with parameters $v, b, r, k, \lambda$, (where $v=b, r=k$ ). Then there exists $a$ b.i.b. design with parameters

$$
v^{\prime}={ }^{\prime} v, \quad b^{\prime}=\left(\begin{array}{l}
v \\
2
\end{array}\right), \quad r^{\prime}=\left(\begin{array}{l}
k \\
2
\end{array}\right), \quad k^{\prime}=\lambda, \quad \lambda^{\prime}=\left(\begin{array}{l}
\lambda \\
2
\end{array}\right)
$$

Theorems 1, 2 are similar to Vanstone's Theorem.

These two theorems will be proved with the aid of a $v \times\left(\begin{array}{l}v \\ 2\end{array}\right)$ matrix $A$ of zeros and ones. Denote the $v$ varieties of the symmetric b.i.b. design by $1,2, \ldots, v$. Label its blocks as $B_{1}, B_{2}, \ldots, B_{v}$. Denote the $\left(\begin{array}{l}v \\ 2\end{array}\right)$ columns of $A$ by the $\left(\begin{array}{l}v \\ 2\end{array}\right)$ pairs $(i j), i<j$ and $i, j=1,2, \ldots, v$. The position of $A$ at the intersection of its $p$ th row and $(i j)$ th column will be called the $(p,(i j))$ position of $A$.

Proof of Theorem 1. We shall construct an incidence matrix $A$ of the second b.i.b. design in Theorem 1 .

Let the entry of $A$ in its $(p,(i j))$ position be 1 if the block $B_{p}$ contains one and only one of the varieties $i, j$ and 0 otherwise. We get a $v \times\left(\begin{array}{l}v \\ 2\end{array}\right)$ matrix $A$ with 0,1 as entries.

Consider the $(i j)$ th column of $\boldsymbol{A}$. In the symmetric b.i.b. design there are $r-\lambda$ blocks each of which contains variety $i$ but not variety $j$. Similarly there are $r-\lambda$ other blocks each of which contains variety $j$ but not variety $i$. The rest of the blocks either contain both the varieties $i, j$ or neither. So the sum of the entries in the (ij)th column of $A$ is $2(r-\lambda)$.

Consider the $p$ th and $q$ th rows of $A, p \neq q$. The two entries at the $(p,(i j))$ and $(q,(i j))$ positions of $A$ are both 1 in the following cases: (i) Block $B_{p}$ contains one of the varieties $i, j$ and the block $B_{q}$ contains the other. (ii) One of the varieties $i, j$ occurs in both $B_{p}$ and $B_{q}$ and the other does not occur in $B_{p}$ and $B_{q}$. 
Next, there are $r-\lambda$ varieties which occur in $B_{p}$, none of which occurs in $B_{q}$. Similarly there are $r-\lambda$ other varieties which occur in $B_{q}$, none of which occurs in $B_{p}$. So corresponding to case (i), there are $(r-\lambda)^{2}$ columns, the intersections of each of which with the $p$ th and $q$ th rows of $A$ have 1 as entries.

There are $\lambda$ varieties common to both $B_{p}$ and $B_{q}$ and $v-2(r-\lambda)+\lambda$ varieties, none of which occurs in $B_{p}$ or $B_{q}$. So corresponding to case (ii), we have $\lambda(v-2 r+\lambda)$, columns, intersections of each of which with the $p$ th and $q$ th rows have respectively entries 0,0 or 0,1 or 1,0 .

It follows that the scalar product of the $p$ th and $q$ th rows of $A$ is $(r-\lambda)^{2}+$ $\lambda(v-2 r+\lambda)=(r-\lambda)(2 r-2 \lambda-1)$ because of the relation $\lambda(v-1)=r(r-1)$.

From a known result, it follows that the row sums of $A$ are constant. This constant is

$$
\left(\begin{array}{l}
v \\
2
\end{array}\right) 2(r-\lambda) / v=(v-1)(r-\lambda)=r(v-r)
$$

Consequently $A$ is an incidence matrix of the second b.i.b. design given in the theorem. Q.E.D.

Proof of Theorem 2. As above, we construct a $v \times\left(\begin{array}{l}v \\ 2\end{array}\right)$ incidence matrix $A$ as follows. This time the entry at the $(p,(i j))$ position of $A$ is 1 if neither variety $i$ nor variety $j$ occurs in the block $B_{p}$.

It is easily seen that there are $v-2(r-\lambda)+\lambda$ blocks not containing any of the varieties $i, j$. So each column sum of $A$ is $v-2 r+\lambda$.

Easily there are $v-2 r+\lambda$ varieties, none of which occurs in $B_{p}$ or $B_{q}$. So there are $\left(\begin{array}{c}v-2 r-\lambda \\ 2\end{array}\right)$ columns each of whose intersections with the $p$ th and $q$ th rows of $A$ contain 1 . In all other columns, the entries in the $p$ th and $q$ th rows are respectively 0,0 or 0,1 or 1,0 . So the scalar product of the $p$ th and $q$ th rows of $A$ is $\left(\begin{array}{c}v-2 r+\lambda \\ 2\end{array}\right)$ and hence of any two rows of $A$.

We now infer that the sum of entries in any row of $A$ is $\left(\begin{array}{l}v \\ 2\end{array}\right)(v-2 r+\lambda) / v=$ $(v-1)(v-2 r+\lambda) / 2=\left(\begin{array}{c}v-r \\ 2\end{array}\right)$ by virtue of the relation $\lambda(v-1)=r(r-1)$.

Thus $A$ is an incidence matrix of the second b.i.b. design in Theorem 2. Q.E.D.

The incidence matrix $A$ of the second b.i.b. design in Vanstone's Theorem has in its $(p,(i j))$ position 1 if both the varieties, $i, j$ occur in the block $B_{p}$ and 0 otherwise. 
3. B.i.b. designs obtainable from two b.i.b. designs. In some cases it is possible to construct a new b.i.b. design from two b.i.b. designs provided their parameters satisfy certain conditions.

As before, designate the varieties by 1,2 etc. and number the block 1,2 etc. Let $A=\left(a_{i j}\right)$ be an incidence matrix of the first b.i.b. design in Theorem 3 so that $a_{i j}=1$ of 0 according as variety $i$ appears in the $j$ th block of this design or not.

Similarly let $B$ be an incidence matrix of the second b.i.b. design in the theorem. Note that $B$ is a $k \times b^{\prime}$ matrix of zeros and ones. Let $\beta_{1}, \beta_{2}, \ldots, \beta_{k}$ be the row vectors of $B$. Then $\beta_{1}+\beta_{2}+\cdots+\beta_{k}=\left(k^{\prime}, k^{\prime}, \ldots, k^{\prime}\right)=$ a row vector with $b^{\prime}$ components.

We shall now construct a $v \times b b^{\prime}$ matrix $C$ with 0 and 1 as entries which will be an incidence matrix of the third b.i.b. design in Theorem 3 .

In each column of $A$, replace the $k$ ones by the $k$ vectors $\beta_{1}, \beta_{2}, \ldots, \beta_{k}$ respectively and $v-k$ zeros by $b^{\prime}$-vectors $(0,0, \ldots, 0)$ respectively. The $v \times b b^{\prime}$ matrix so obtained is the desired matrix $C$.

For, it is easily seen that the sum of the entries in any column of $C$ is $k^{\prime}$. The scalar product of any two rows of $C$ is $\lambda^{\prime}$. Then the sum of the entries in any row of $C$ is independent of the row and is $b b^{\prime} k^{\prime} / v=b k r^{\prime} k^{\prime} /\left(v k^{\prime}\right)=r r^{\prime}$. As a consequence, $C$ is an incidence matrix of the third b.i.b. design in Theorem 3 and this completes the proof of this theorem.

Given a positive integer $k>2$. There exists a trivial b.i.b. design with parameters $v^{\prime}=k, b^{\prime}=k, k^{\prime}=k-1, r^{\prime}=k-1, \lambda^{\prime}=k-2$. Using this b.i.b. design as the second b.i.b. design in Theorem 3 we get the second b.i.b. design in Theorem 4. This proves Theorem 4.

Theorem 4 was proved by Das and Kulshreshtha [2] under the redundant restriction that the first design is obtained by the method of difference.

We now prove Theorem 5. Its proof is based on the same ideas as in the proof of Theorem 4 .

Let $A=\left(a_{i j}\right)$ be an incidence matrix of the first resolvable b.i.b. design in the theorem so that $a_{i j}=1$ or 0 according as variety $i$ appears in the $j$ th block of the design or not. We may suppose that the first set of $n$ blocks of this design contain one complete replication of the $v$ varieties, the second set of $n$ blocks contain a complete replication and so on.

Similarly let $B=\left(b_{i j}\right)$ be an incidence matrix of the second resolvable b.i.b. design in the theorem. Again we suppose that the first set of $n^{\prime}$ blocks of this design contain a complete replication of its $k$ varieties, the second set of $n^{\prime}$ blocks contain another complete replication and so on. Let $\beta_{1}, \beta_{2}, \ldots, \beta_{k}$ be the row vectors of $B$.

In each column of $A$, replace the $k$ ones by $\beta_{1}, \beta_{2}, \ldots, \beta_{k}$ respectively and the $v-k$ zeroes by $v-k b^{\prime}$-vectors $(0,0, \ldots, 0)$. We thus get a $v \times b b^{\prime}$ matrix $C$ of zeros and ones. 
If we add the $v$ rows of $C$, we get the $b b^{\prime}$-vector $\left(k^{\prime}, k^{\prime}, \ldots, k^{\prime}\right)$ since $\beta_{1}+\beta_{2}+\cdots+\beta_{k}=b^{\prime}$-vector $\left(k^{\prime}, k^{\prime}, \ldots, k^{\prime}\right)$. As the scalar product of $\beta_{i}, \beta_{j}, i \neq j$, is $\lambda^{\prime}$ and the scalar product of any two rows of $A$ is $\lambda$, it easily follows that the scalar product of any two rows of $C$ is $\lambda \lambda^{\prime}$ and then any row sum of $C$ is $k^{\prime} b b^{\prime} / v=k^{\prime} v r b^{\prime} /(k v)=r r^{\prime}$.

We note that the first $n n^{\prime}$ column vectors of $C$, on addition, gives a column $n k$-vector (i.e. $v$-vector) of ones. The same holds for the second set of $n n^{\prime}$ columns vectors and so on. We infer that $C$ is an incidence matrix of a resolvable b.i.b. design-the third resolvable b.i.b. design in Theorem 5 . This proves Theorem 5.

If $k$ is an even integer $\geq 4$, one can easily construct a trivial resolvable b.i.b. design with parameters

$$
v^{\prime}=k, \quad b^{\prime}=\left(\begin{array}{c}
k \\
k / 2
\end{array}\right), \quad r^{\prime}=\left(\begin{array}{c}
k-1 \\
(k-2) / 2
\end{array}\right), \quad k^{\prime}=k / 2, \quad \lambda^{\prime}=\left(\begin{array}{c}
k-2 \\
(k-4) / 2
\end{array}\right) .
$$

One merely chooses all possible sets of $k / 2$ varieties from a set of $k$ varieties. Then one pairs the sets-one containing half the varieties and the other containing the rest of the $k / 2$ varieties. A moment's consideration shows that we have constructed a resolvable b.i.b. with the above parameters. Using this resolvable b.i.b. design as the second resolvable b.i.b. design in Theorem 5, we get the second resolvable design in Theorem 6 . This completes the proof of Theorem 6.

\section{REFERENCES}

1. Vanstone, S. A. A note on a construction of b.i.b. d.'s Utilitas Mathematica, Vol. 7 (1975) pp. 321-322.

2. Das, M. N. and Kulshreshtha, A. C. On derivation of initial blocks with more than one initial block The Australian Journal of Statistics Vol. 10, No. 2, August 1968, pp. 75-82.

\footnotetext{
DePaRTMENT OF MATHEMATICS

CONCORDIA UNIVERSTTY (LOYOLA COMPUS)

Montreal, CANADA
} 